Article

\title{
Nitric Oxide Induced st 2 Expression Is Inhibited by the Nitric Oxide Reductase, NorV, in a Clade 8 Escherichia coli O157:H7 Outbreak Strain
}

\author{
Rim Al Safadi ${ }^{\dagger}$, Michelle L. Korir ${ }^{\ddagger}$ (D) and Shannon D. Manning *(D) \\ Department of Microbiology and Molecular Genetics, Michigan State University, East Lansing, MI 48824, USA; \\ rimalsafadi@gmail.com (R.A.S.); mkorir@aurora.edu (M.L.K.) \\ * Correspondence: mannin71@msu.edu \\ + Current Address: Azm Center for Research in Biotechnology and Its Applications, LBA3B, EDST, \\ Lebanese University, Tripoli 1300, Lebanon. \\ $\ddagger$ Current Address: Department of Biology, Aurora University, Aurora, IL 60506, USA.
}

Citation: Al Safadi, R.; Korir, M.L.; Manning, S.D. Nitric Oxide Induced st $x 2$ Expression Is Inhibited by the Nitric Oxide Reductase, NorV, in a Clade 8 Escherichia coli O157:H7 Outbreak Strain. Microorganisms 2022, 10, 106. https://doi.org/10.3390/ microorganisms10010106

Academic Editor: Tim A. McAllister, Flemming Scheutz, Linda Chui, Chad R. Laing, Nicole Van De Kar, Kim Stanford and Patricia Griffin

Received: 3 November 2021 Accepted: 29 December 2021 Published: 5 January 2022

Publisher's Note: MDPI stays neutral with regard to jurisdictional claims in published maps and institutional affiliations.

Copyright: (C) 2022 by the authors. Licensee MDPI, Basel, Switzerland. This article is an open access article distributed under the terms and conditions of the Creative Commons Attribution (CC BY) license (https:// creativecommons.org/licenses/by/ $4.0 /)$.

\begin{abstract}
Escherichia coli O157:H7 pathogenesis is due to Shiga toxin (Stx) production, though variation in virulence has been observed. Clade 8 strains, for instance, were shown to overproduce Stx and were more common among hemolytic uremic syndrome cases. One candidate gene, nor $V$, which encodes a nitric oxide (NO) reductase found in a clade 8 O157:H7 outbreak strain (TW14359), was thought to impact virulence. Hence, we screened for norV in $303 \mathrm{O} 157$ isolates representing multiple clades, examined stx2 expression following NO exposure in TW14359 for comparison to an isogenic mutant $(\Delta$ nor $V)$, and evaluated survival in THP-1 derived macrophages. nor $V$ was intact in strains representing clades 6-9, whereas a 204 bp deletion was found in clades 2 and 3. During anaerobic growth, NO induced st $x 2$ expression in TW14359. A similar increase in st $x 2$ expression was observed for the $\Delta$ nor $V$ mutant in anaerobiosis, though it was not impaired in its ability to survive within macrophages relative to TW14359. Altogether, these data suggest that NO enhances virulence by inducing Stx2 production in TW14359, and that toxin production is inhibited by NorV encoded by a gene found in most clade 8 strains. The mechanism linked to these responses, however, remains unclear and likely varies across genotypes.
\end{abstract}

Keywords: Shiga toxin; Escherichia coli; O157; virulence; pathogenesis; nitric oxide

\section{Introduction}

Shiga toxin-producing Escherichia coli (STEC) O157:H7 is a leading cause of gastrointestinal illness linked to food and waterborne outbreaks worldwide. Patients infected with STEC O157 often present with hemorrhagic colitis, although some can develop hemolytic uremic syndrome (HUS) [1] that can lead to kidney failure and death. In the U.S., an annual $46.2 \%$ hospitalization rate and $0.5 \%$ death rate were reportedly attributable to STEC O157 [2], while the incidence of all STEC infections was estimated to be 5.9 cases per 100,000 individuals in 2018 [3]. Considerable variation in the number of infections, however, has been documented across different regions in the U.S. [4]

STEC pathogenesis is mainly due to the production of one or more Shiga toxins (Stx) encoded by genes carried on lambda-like bacteriophages [5]. Other virulence factors are also important. For instance, STEC strains possessing genes like eae (intimin) found on the locus of enterocyte effacement (LEE) pathogenicity island [6], contribute to attaching and effacing lesion formation on intestinal epithelial cells. E. coli strains possessing stx as well as the LEE island are classified as enterohemorrhagic E. coli. (EHEC), which were found to be more virulent than STEC strains lacking these factors [7]. Furthermore, EHEC strains producing the Stx2a and/or Stx2c variants were observed to be more virulent causing a higher frequency of HUS than strains producing the Stx1 variant alone or with 
Stx2a [5,8,9]. Differences have been reported across populations, however, indicating that strain specific factors are also important for clinical phenotypes. Indeed, we previously showed that a diverse set of $\mathrm{O} 157$ strains could be classified into nine distinct clades based on the phylogenetic analysis of 96 single nucleotide polymorphisms (SNPs) [10]. Most importantly, strains belonging to clade 8 were significantly more common among HUS cases and more frequently possessed genes encoding Stx2a and/or Stx2c compared to other clades. Follow up studies showed that clade 8 strains also had greater $s t x 2$ expression levels following exposure to epithelial cells [11] and more than the expected number of Stx-encoding bacteriophage insertion sites occupied by prophages lacking stx [12]. Notably, deletion of a non-Stx-encoding prophage in a high stx2a-expressing clade 8 strain (TW14313) drastically reduced st $x 2 a$ expression levels in the mutant relative to the wild type [12]. Taken together, these data suggest that clade 8 strains possess unique traits that may enhance their ability to cause more severe disease.

A genome analysis of the clade 8 O157:H7 strain (TW14359), which was implicated in the 2006 North American spinach outbreak that caused a higher-than-average frequency of HUS [13], uncovered several traits that could promote virulence [14]. One candidate gene, nor $V$, encodes a nitric oxide (NO) reductase (NorV) that detoxifies NO in oxygen limiting conditions [15]. Two versions of nor $V$ have been identified [14]. An intact functional norV was detected in TW14359 [14], while a non-functional copy containing a 204 bp deletion was found in the EDL933 (clade 3) [16] and the Sakai (clade 1) [17] O157:H7 outbreak strain genomes [14]. An initial screen of 100 O157:H7 strains recovered from multiple sources detected the $204 \mathrm{bp}$ deletion in $58 \%$ of strains and a correlation with stx1 [14], which we found to be overrepresented among strains representing clades 1-3 [10]. These findings indicate that nor $V$ functionality and its impact on virulence may be restricted to strains with a specific genetic background.

A prior study also demonstrated that possession of an intact nor $V$ protected different EHEC O157:H7 strains from growth inhibition by NO under anaerobic conditions unlike strains possessing the non-functional version of nor $V$ [18]. Additionally, mutants lacking nor $V$ had decreased survival within macrophages. Since macrophage uptake and the subsequent production of $\mathrm{NO}$ are a critical part of the innate immune response to bacterial pathogens, these data suggest that possession of a functional nor $V$ can protect a bacterium from macrophage killing. Indeed, the same study found that insertion of a functional norV into the EDL933 chromosome subsequently increased st 2 expression in the presence of NO inside macrophages [18]. As these data were generated with a strain that was not classified as clade 8, the function of nor $V$ in a more virulent strain background is not known. Consequently, the goal of this study was to examine the effects of $\mathrm{NO}$ and nor $V$ mutagenesis on growth, st 2 expression, and macrophage survival using the clade 8 spinach outbreak strain, TW14359.

\section{Materials and Methods}

\subsection{Bacterial Strains, norV Detection, and Growth Conditions}

The E. coli O157:H7 spinach outbreak strain, TW14359, was used for all experiments; it possesses $s t x 2 a$ and $s t x 2 c$ and was previously classified as clade 8 by SNP genotyping [10]. This strain was previously recovered by the Michigan Department of Health and Human Services (MDHHS) from a patient who had consumed spinach linked to the national 2006 outbreak [13]. An additional set of 303 previously characterized 0157 strains with seven stx profiles representing the predominant clades 1-3 and 6-9 [10], was screened for the presence of an intact (functional) or deleted version of norV. These strains were recovered from clinical cases in Michigan between 2001 and 2006 as described [19] Differences in the frequencies of stx profiles and nor $V$ type were detected using the likelihood chi-square test or Fisher's exact test for sample sizes less than five.

After overnight growth in Luria-Bertani (LB) broth at $37^{\circ} \mathrm{C}$ and DNA isolation, PCR was performed to detect the presence of a functional nor $V$ in all 303 strains. The primer set norV698_F and norV1373_R, which targets the region flanking the norV deletion (Table 1), 
was used with the following PCR conditions: $10 \mathrm{~min}$ at $94{ }^{\circ} \mathrm{C}$ plus 30 cycles of $1 \mathrm{~min}$ at $92{ }^{\circ} \mathrm{C}, 30 \mathrm{~s}$ at $53{ }^{\circ} \mathrm{C}$ and $1 \mathrm{~min}$ at $72{ }^{\circ} \mathrm{C}$ followed by $20 \mathrm{~min}$ at $72{ }^{\circ} \mathrm{C}$.

Table 1. Oligonucleotide primers developed for use in this study.

\begin{tabular}{|c|c|c|c|}
\hline Primer & Sequence $\left(5^{\prime}-3^{\prime}\right)$ & Target & Amplicon Size \\
\hline $\begin{array}{c}\text { norv698_F } \\
\text { norv1373_R }\end{array}$ & $\begin{array}{l}\text { ATAACCCGACGCAAATTGT } \\
\text { TATCCGGGACTTCACTCCA }\end{array}$ & nor $V$ & $\begin{array}{c}675 \mathrm{bp} \text { (intact) } \\
470 \mathrm{bp} \text { (deletion) }\end{array}$ \\
\hline $\begin{array}{l}\text { norV-del1 } \\
\text { norV-del2 }\end{array}$ & $\begin{array}{c}\text { GCAATTAGCAAGA } \\
\text { CATCTTTTTAGAACACGCTGAA } \\
\text { TAAATTGAGGTTGCTGTG } \\
\text { TAGGCTGGAGCTGCTTC } \\
\text { CACCAGTTGGCGGGC } \\
\text { GGCGAAGCCCGAACCA } \\
\text { ATGATCACAATGCCGTTGC } \\
\text { CATATGAATATCCTCCTTAG }\end{array}$ & $\begin{array}{c}\text { Kanamycin marker } \\
\text { flanked by } 50 \text { bp of nor } V\end{array}$ & $1577 \mathrm{bp}$ \\
\hline $\begin{array}{l}\text { norV-del3 } \\
\text { norV-del4 }\end{array}$ & $\begin{array}{l}\text { GATATTCGCCAGCACATCAA } \\
\text { AAACTGCTCGGCAAATTCAC }\end{array}$ & $\Delta$ nor $V$ & $\begin{array}{c}1868 \text { bp (TW14359) } \\
1905 \text { bp ( } \Delta \text { nor } V)\end{array}$ \\
\hline $\begin{array}{l}\text { norvcompl_F } \\
\text { norvcompl_R }\end{array}$ & $\begin{array}{c}\text { AGATATTGTCATATCGACCATTGGA } \\
\text { AATGATCACAATGCCGTTGC }\end{array}$ & $\begin{array}{l}\text { nor } V+\text { flanking } \\
\text { region and nor } V \text { promoter }\end{array}$ & $1620 \mathrm{bp}$ \\
\hline
\end{tabular}

\subsection{Construction of a norV Mutant}

nor $V$ was deleted from the chromosome of wild-type (WT) strain TW14359 using the red recombination procedure [20]. Briefly, recombinant PCR products containing a kanamycin $(\mathrm{Km})$ resistance marker flanked by $50 \mathrm{bp}$ sequences homologous to the upstream and downstream regions of nor $V$ were generated from plasmid pKD4 [21,22] using primers norv-del1/del2 (Table 1). PCR products were electroporated into the red recombinaseproducing WT strain containing pKM208 as described previously [23]. Transformants were identified by growth on LB agar supplemented with $25 \mu \mathrm{g} / \mathrm{mL} \mathrm{Km}$ following incubation at $37^{\circ} \mathrm{C}$. The deletion in nor $V(\Delta n o r V)$ was confirmed by PCR-based restriction fragment length polymorphism using the norv-del3/del4 primer set followed by digestion with PstI for $2 \mathrm{~h}$ at $37^{\circ} \mathrm{C}$.

For complementation of $\Delta n o r V$, a 1620 bp region containing the nor $V$ coding region plus additional flanking regions including the nor $V$ promoter, were amplified by PCR from strain TW14539 genomic DNA using TaKaRa LA Taq polymerase (Takara Bio; Madison, WI, USA) with the norv-compl_F/norvcompl_R primer set. The resulting PCR products were cloned into the pCR2.1-TOPO vector (Invitrogen; Carlsbad, CA, USA) to construct the pCR2.1-TOPOnor $V$ plasmid. This plasmid was transformed into the $\Delta$ nor $V$ strain, creating the $\Delta$ nor $V:: n$ or $V_{\mathrm{WT}}$ strain. Next, we electroporated the $\mathrm{PCR} 2.1-\mathrm{TOPO}$ empty vector into the $\Delta$ nor $V$ strain, which resulted in a $\Delta$ nor $V::$ vector strain for use as a control. Transformants were grown overnight in LB supplemented with $100 \mu \mathrm{g} / \mathrm{mL}$ ampicillin at $37^{\circ} \mathrm{C}$.

\subsection{Bacterial Gene Expression}

Prior to RNA extraction, the WT and mutant strains were grown for 6 and $24 \mathrm{~h}$ in LB broth at $37^{\circ} \mathrm{C}$ in the presence or absence of $200 \mu \mathrm{M}$ of the nitric oxide donor (NOR4) (Sigma-Aldrich; Burlington, MA, USA). The concentration of NO was determined using the Nitrate/nitrite Colorimetric Assay Kit (Sigma-Aldrich). Strains were cultivated at $37^{\circ} \mathrm{C}$ with shaking (aerobic) or without shaking in the presence of $5 \% \mathrm{CO}_{2}$ (anaerobic). cDNA was synthesized from the extracted RNA by reverse transcription as described previously [24]; three independent RNA populations were extracted per sample. Quantitative real-time PCR (qRT-PCR) was performed utilizing amplification conditions and primers specific for the B subunit gene of stx2, that differentiates between st $x 2 a$ and st $x 2 c$ as was carried out previously $[11,24]$. Expression data are reported for "stx2" for simplicity. For normalization, 
published conditions and primers were used to amplify the 16S rRNA gene, $r$ rsH [24]. Transcription differences were quantified as described [11,22], and expression levels were presented for strain TW14359 relative to $\Delta n o r V$ or the same strain grown in different conditions (e.g., aerobic vs. anaerobic). Fold change in $s t \times 2$ expression was calculated using the ddCt method [25]; a fold change of $\geq 2$ was considered biologically significant.

\subsection{Cell Culture and EHEC Infection}

THP-1 monocyte-like cells (ATCC TIB-202) were cultured in Roswell Park Memorial Institute 1640 (RPMI) growth medium (Gibco; Amarillo, TX, USA) supplemented with $2 \mathrm{mM}$ L-glutamine (Gibco), 10\% fetal bovine serum (FBS; Atlanta Biologicals; Minneapolis, $\mathrm{MN}, \mathrm{USA}$ ), and $1 \%$ penicillin/streptomycin (Gibco) at $37^{\circ} \mathrm{C}$ with $5 \% \mathrm{CO}_{2}$. Cells were differentiated into macrophages by incubation with $100 \mathrm{nM}$ phorbol 12-myristate 13-acetate (PMA; Sigma-Aldrich, St. Louis, MO, USA) in RPMI medium with 2\% FBS for $24 \mathrm{~h}$ as was described [26]. Using a published protocol [27] that was modified for EHEC O157:H7, the cells were seeded at a density of $1 \times 10^{6}$ cells per well in 24 well plates. Bacteria were added to the cell monolayer at a multiplicity of infection (MOI) of 10 and incubated at $37^{\circ} \mathrm{C}$ with $5 \% \mathrm{CO}_{2}$ for $1 \mathrm{~h}$. The cells were washed with phosphate-buffered saline (PBS) and fresh medium containing $100 \mathrm{mg} / \mathrm{mL}$ of gentamicin was added for $1 \mathrm{~h}$ to kill extracellular bacteria. The infected monolayers were lysed by adding $0.1 \%$ triton X-100 (Sigma-Aldrich) in PBS. Lysates were diluted and plated onto LB agar and incubated at $37^{\circ} \mathrm{C}$ overnight to quantify colony forming units (CFUs). The number of intracellular (phagocytosed) bacteria was determined by normalizing to the total number of bacteria left in the well after the $1 \mathrm{~h}$ infection period. The percent survival was calculated by dividing the number of intracellular bacteria at $24 \mathrm{~h}$ by the total number of intracellular bacteria $1 \mathrm{~h}$ after adding antibiotics and multiplying by 100 . A Student's t-test was used to determine if there were significant differences between strains as measured using the mean; a $p$-value $\leq 0.05$ was considered significant.

\section{Results}

\section{1. norV Distribution by Clade and Association with Stx Gene Profiles}

To detect the presence of the intact (functional) and deleted nor $V$ variants, all $303 \mathrm{O} 157$ clinical isolates were examined. A $470 \mathrm{bp}$ product representing the presence of a nonfunctional nor $V$ containing a 204 bp deletion was found in 197 (65.0\%) strains. By contrast, a $675 \mathrm{bp}$ product representing the intact and functional version of nor $V$ was found in the remaining 106 (35.0\%) strains. Differences in the distribution of the intact nor $V$ were observed across the O157 clades previously classified using SNP genotyping. [10]. Among the predominant $\mathrm{O} 157$ clades examined, the non-functional nor $V$ containing the deletion was detected only in strains belonging to clades 1-3, while the intact nor $V$ was detected in all strains representing clades 6-9 (Figure 1). Among those 197 strains containing the 204 bp deletion, most $(83.2 \%$ ) belonged to clade 2 , the predominant lineage examined in this subset of clinical strains. Comparatively, most of the strains containing the intact norV belonged to clades 8 and 7 .

The non-random distribution of the two norV variants across the SNP-based phylogeny [10] was also linked to the distribution of stx subtypes. The 303 strains evaluated had seven different stx profiles that varied by clade and norV type (Table 2). Strains with the intact, functional nor $V$ possessed all seven stx variants, though some were more common than others. Strains with an intact nor $V$, for instance, were significantly more likely to have either st $x 2$ alone or with $s t x 2 c$ compared to the remaining strains with an intact nor $V$ as well as those with a deleted version (Fisher's exact test $p \leq 0.0001$ and $p \leq 0.0001$, respectively). Of the 81 strains containing st $x 2$ only $(n=41)$ or st $x 2$ with $\operatorname{st} x 2 c(n=40)$, most $(n=63)$ belonged to clade 8 . By contrast, strains with the deleted version of nor $V$ were significantly more likely to possess both st $x 1$ and st $x 2$ relative to all other strains (Fisher's exact test $p \leq 0.0001)$. Among the 186 strains with st $x 1$ and st $x 2$, most belonged to clades $2(n=152)$ and $3(n=32)$. 


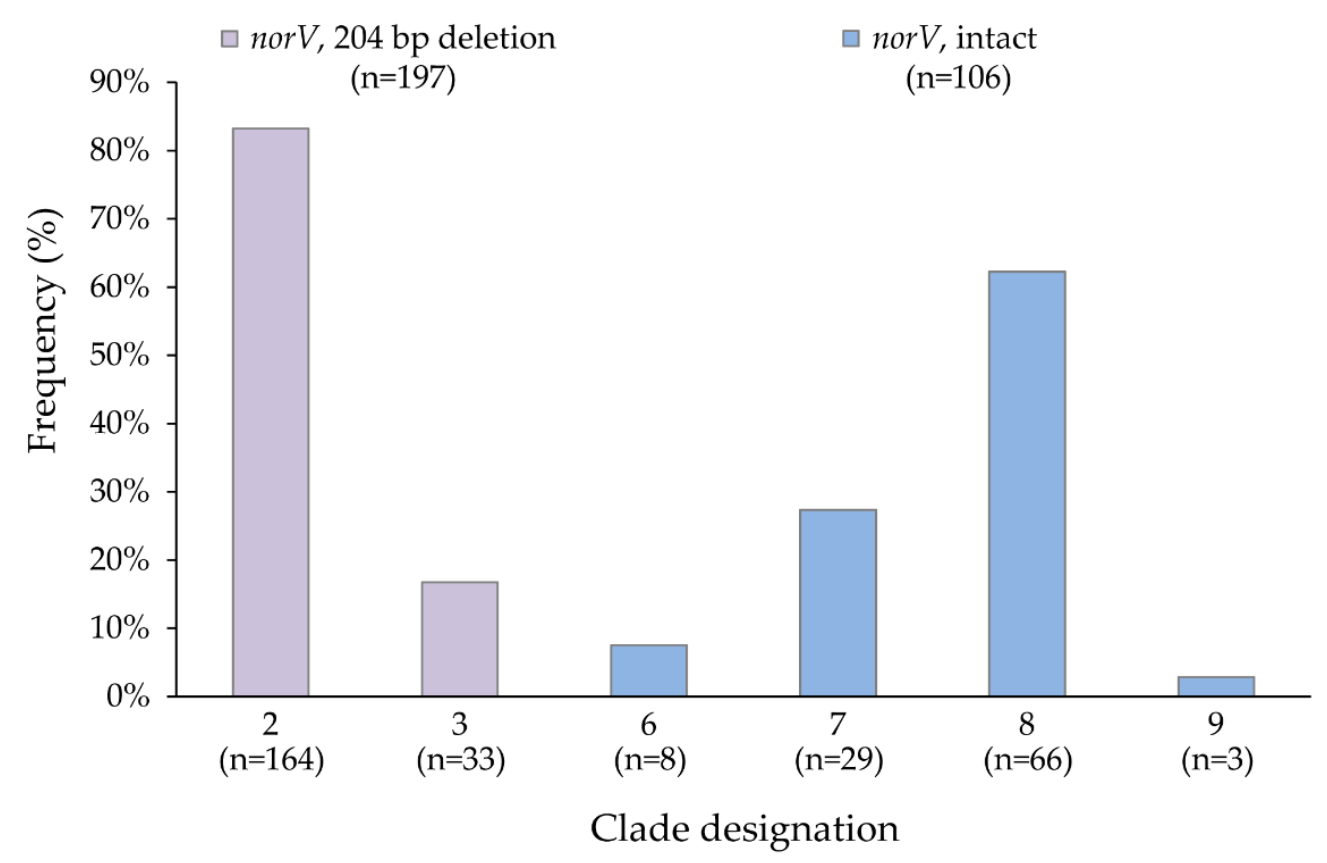

Figure 1. Distribution and percent of nor $V$ variants among 303 Shiga toxin-producing Escherichia coli O157 clinical isolates. Percentages for clades 2 and 3 were calculated using the total number of isolates with a deletion in $\operatorname{nor} V(n=197$, purple bars) as the denominator, whereas clades $6-9$ used the total number with an intact $n o r V(n=106$, blue bars) as the denominator.

Table 2. Distribution of stx profiles by clade and norV type among 303 Shiga toxin-producing Escherichia coli $\mathrm{O} 157$ clinical isolates.

\begin{tabular}{cccccccccc}
\hline Clade & norV + & stx1 & $\begin{array}{c}\text { stx1, } \\
\text { stx2 }\end{array}$ & $\begin{array}{c}\text { stx1, stx2, } \\
\text { stx2c }\end{array}$ & $\begin{array}{c}\text { stx1, } \\
\text { stx2c }\end{array}$ & stx2 & $\begin{array}{c}\text { stx2, } \\
\text { stx2c }\end{array}$ & stx2c & Total * \\
\hline 2 & Deletion & $2(1.2)$ & $152(92.7)$ & - & - & $10(6.1)$ & - & - & 164 \\
\hline 3 & Deletion & $1(3.0)$ & $32(97.0)$ & - & - & - & - & - \\
\hline 6 & Intact & - & - & - & - & $1(12.5)$ & $1(12.5)$ & $6(75.0)$ & 8 \\
\hline 7 & Intact & $1(3.5)$ & - & $1(3.5)$ & $7(24.1)$ & - & $6(20.7)$ & $14(48.3)$ & 29 \\
\hline 8 & Intact & - & $1(1.5)$ & $1(1.5)$ & - & $30(45.5)$ & $33(50.0)$ & $1(1.5)$ & 66 \\
\hline 9 & Intact & - & $1(33.3)$ & - & $2(66.7)$ & - & - & - & 3 \\
\hline
\end{tabular}

* Percentages were calculated using the total number of isolates per clade as the denominator. + Differences in the frequencies of stx profiles by nor $V$ type were detected using the likelihood chi-square test; the Fisher's exact test was used for sample sizes less than five.

\subsection{Impact of NO on stx2 Expression in the Clade 8 Outbreak Strain}

To determine whether strain TW14359 had higher stx2 expression in response to NO, the strain was grown in LB with and without the NOR4 nitric oxide donor. In aerobic conditions, the stx 2 expression levels were not significantly altered in the presence or absence of NO after $6 \mathrm{~h}$ of growth (data not shown). The same was true in anaerobic conditions after $6 \mathrm{~h}$. Increasing the incubation time, however, was associated with an increase in stx 2 expression but only in anaerobic conditions. Specifically, after $24 \mathrm{~h}$ of anaerobic growth, stx 2 expression was 2.2-fold higher in the presence of NO relative to the absence of NO (Figure 2). No significant difference was observed for aerobic conditions after $24 \mathrm{~h}$ of growth, indicating that $\mathrm{NO}$ activates stx 2 expression in the clade 8 spinach outbreak strain only in anaerobic conditions. 


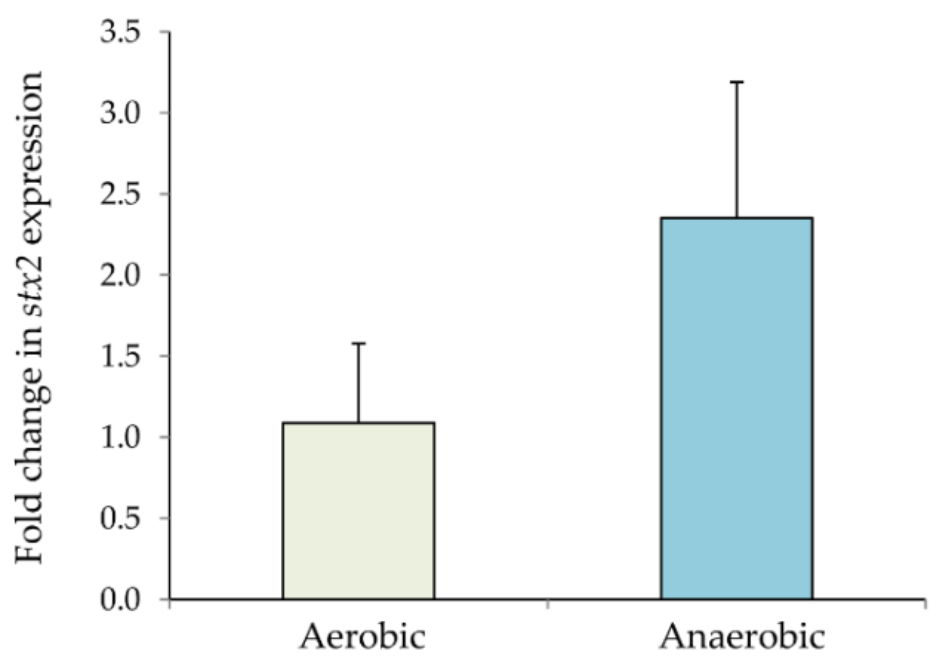

Figure 2. Fold change in st $x 2$ gene expression in the E. coli O157:H7 outbreak strain, TW14359, in the presence of $\mathrm{NO}$ in aerobic and anaerobic conditions after $24 \mathrm{~h}$ of growth. The st $x 2$ transcripts were normalized to the $16 \mathrm{~S}$ rRNA transcripts and are shown relative to the level of transcription in the absence of NO. The means of at least three independent experiments are shown by growth condition and the bars represent the standard deviation of the means. A fold-change $\geq 2$ was considered biologically significant.

\subsection{Role of norV in stx2 Expression in the Clade 8 Outbreak Strain across Growth Conditions}

Since nor $V$ contributes to $\mathrm{NO}$ detoxification and an intact nor $V$ was present in all 66 clade 8 strains examined, we constructed an isogenic non-polar null nor $V$ mutant $(\Delta n o r V)$ in the wild-type (WT) spinach outbreak strain TW14359. stx2 expression was quantified in $\Delta$ nor $V$ relative to the WT strain in the presence and absence of NO in both aerobic and anaerobic conditions following $24 \mathrm{~h}$ of growth. Under aerobic conditions, stx 2 transcription levels were similar between the WT and $\Delta$ norV in the presence and absence of $\mathrm{NO}$ (Figure 3A). As compared to the WT strain, stx2 transcription levels were $1.0 \pm 0.3$ in the absence of $\mathrm{NO}$ and $1.7 \pm 0.6$ with $\mathrm{NO}$. This finding suggests that nor $V$ does not affect st $x 2$ expression in aerobic conditions regardless of $\mathrm{NO}$ availability. By contrast, growth in anaerobic conditions for $24 \mathrm{~h}$ showed a difference in st $x 2$ expression in the presence of $\mathrm{NO}$ (Figure 3B). Specifically, the mutant $\Delta$ nor $V$ strain had a 3.2-fold increase in stx2 expression as compared to the WT strain when NO was present. The WT and $\Delta n o r V$ revealed similar transcript levels of $s t x 2$ in the absence of $\mathrm{NO}$, suggesting that nor $V$ inhibits $s t x 2$ expression solely in the presence of NO.

Next, we complemented the nor $V$ deletion in trans by expressing the WT nor $V$ on the pCR2.1-TOPO plasmid in the $\Delta$ nor $V$ strain $\left(\Delta\right.$ nor $\left.V:: n o r V_{\mathrm{WT}}\right)$. norV expression in the complemented $\Delta$ nor $V:: n o r V_{\text {WT }}$ strain was confirmed by qPCR, though the level of norV transcription was higher in the complemented strain than the WT. We then compared stx 2 expression levels in the WT, $\Delta$ nor $V$, and $\Delta$ nor $V:: n$ or $V_{\mathrm{WT}}$ strains as well as the $\Delta$ nor $V$ strain containing the empty vector ( $\Delta$ nor $V:: v e c t o r)$ using the same conditions. Increased expression in stx2 was observed in the $\Delta n o r V$ strain relative to the WT (data not shown), yet a similar increase was observed in both the $\Delta$ nor $V:: n$ or $V_{\mathrm{WT}}$ and $\Delta$ nor $V::$ vector strains.

\subsection{Role of norV in Uptake and Survival of the Clade 8 Outbreak Strain within Macrophages}

To determine how nor $V$ impacts phagocytic uptake and intracellular survival of TW14359, we infected human THP-1-derived macrophages with the WT and isogenic $\Delta$ nor $V$ strains. At $1 \mathrm{~h}$ post infection, no difference in phagocytic uptake was observed between the WT and $\Delta$ norV strains (Figure 4A). Specifically, $0.31 \% \pm 0.04$ of the total WT cells per well and $0.34 \% \pm 0.06$ of $\Delta$ nor $V$ cells were taken up by the THP-1 macrophages after $1 \mathrm{~h}$. Enhanced survival within THP-1 macrophages, however, was observed for the 
$\Delta$ nor $V$ strain $24 \mathrm{~h}$ post infection relative to the WT (Figure 4B). Nonetheless, this difference was not statistically significant (Student's $t$-test $p=0.10$ ). After normalizing to initial uptake levels, the percent survival of the WT and $\Delta$ nor $V$ strains within macrophages was $2.9 \% \pm 0.7$ and $4.1 \% \pm 0.7$, respectively.

A)

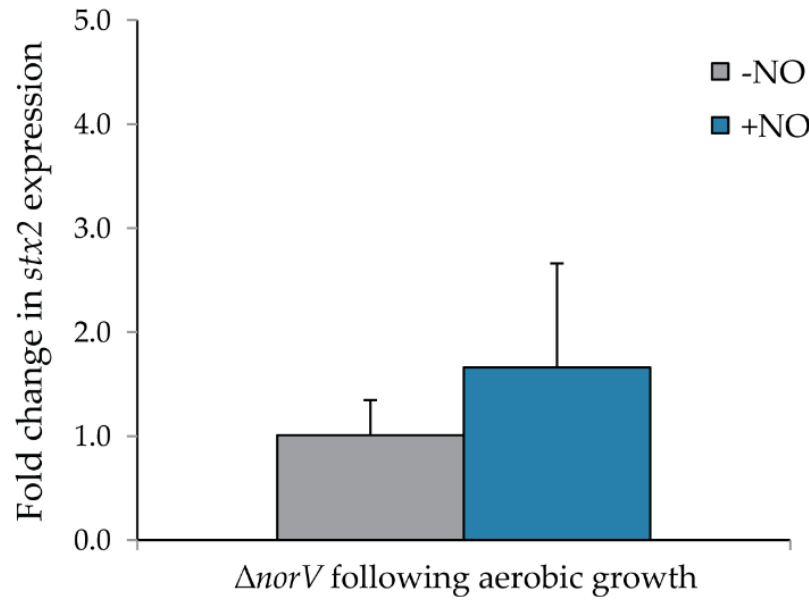

B)

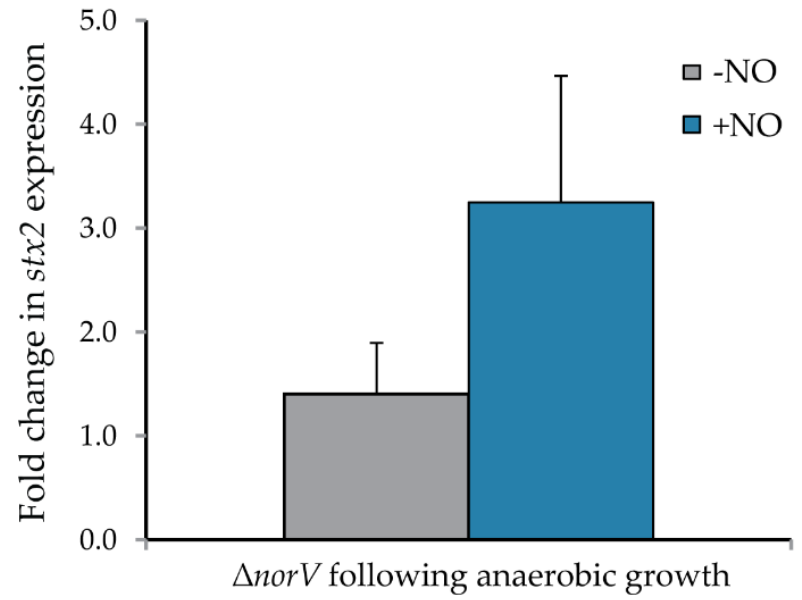

Figure 3. Fold change in stx2 gene expression in (A) aerobic and (B) anaerobic conditions with and without nitric oxide (NO) in the null $\Delta$ nor $V$ mutant strain relative to the wild-type (WT) TW14359 O157:H7 strain. The stx2 transcripts in each condition were normalized to the amount of $16 \mathrm{~S}$ transcripts and are shown relative to the level of transcription in the WT for each growth condition. The boxes represent the means of at least three independent experiments and the bars are the standard deviation of the means. A fold-change $\geq 2$ was considered biologically significant.

An examination of stx 2 expression in the WT versus $\Delta$ nor $V$ strain following infection of THP-1 macrophages showed no significant difference in expression levels $1 \mathrm{~h}$ post infection (Student's $t$-test $p=0.12$ ). On average, the $\Delta$ nor $V$ strain had a 1.4-fold decrease in st $x 2$ expression relative to the WT. Expression of st $x 2$ at $24 \mathrm{~h}$ post infection could not be quantified for either strain as the number of transcripts was below the level of detection.

A)

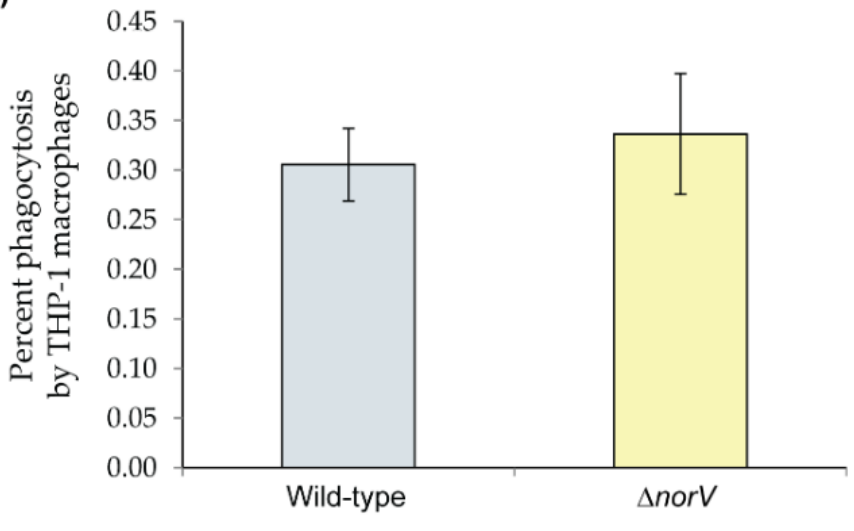

B)

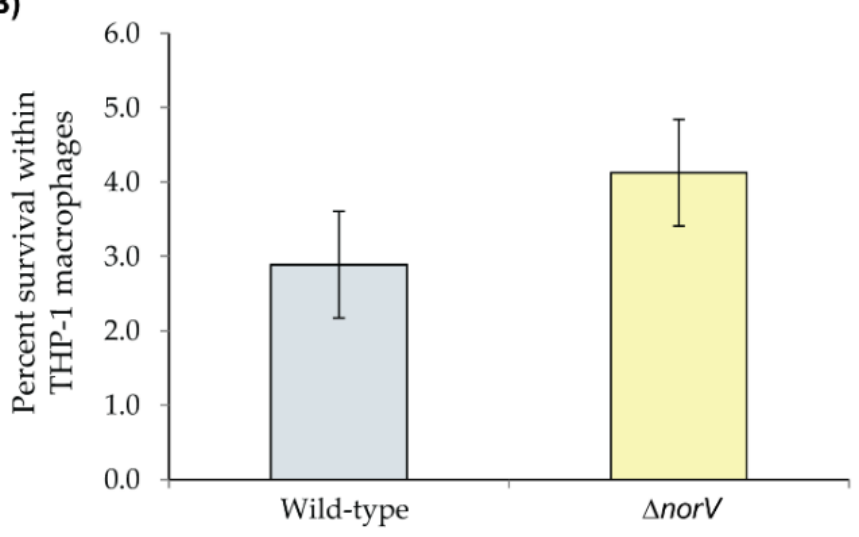

Figure 4. (A) Phagocytic uptake and (B) intracellular survival of the E. coli O157:H7 wild-type (WT) TW14359 outbreak strain relative to the isogenic $\Delta$ nor $V$ strain in THP-1 macrophages. Uptake was examined $1 \mathrm{~h}$ post-infection and survival was determined after $24 \mathrm{~h}$. Boxes represent the means of three independent replicates and the bars show the standard deviation of the means. No significant differences were observed between strains for either phagocytic uptake or intracellular survival using a Student's $t$-test. 


\section{Discussion}

EHEC O157 strains belonging to the clade 8 lineage were shown to be more commonly isolated from HUS cases than other lineages [10] and have an enhanced ability to adhere to epithelial cells and express $s t x 2$ and other key virulence genes [11]. These findings suggest that clade 8 strains may be more virulent than strains representing other $\mathrm{O} 157$ lineages. Indeed, a prior study demonstrated enhanced virulence of the clade 8 spinach outbreak strain, K3995, relative to other O157:H7 strains in both rabbits and mice, which was due to increased Stx2 production [28]. This 2006 multistate spinach outbreak also contributed to a higher rate of HUS than has been observed for other O157:H7 outbreaks [13]. Additionally, a genome sequence comparison between the clade 8 spinach outbreak strain, TW14359, and two O157:H7 outbreak strains that caused low HUS case rates, found norV to be unique to the clade 8 strain [14]. Our evaluation of $303 \mathrm{O} 157$ isolates, which represented the major phylogenic lineages previously defined by SNP genotyping [10], showed that $65 \%$ possess a $204 \mathrm{bp}$ deletion in nor $V$ and that all of these strains belong to clades 2 and 3 . The genome sequence of the EDL933 outbreak strain representing clade 3 also shows a deletion in norV [16] as does the clade 1 strain, RIMD 050995, linked to a 1996 outbreak in Sakai Japan [17]. Comparatively, the intact, functional version of nor $V$ was found in $35 \%$ of the 303 O157 strains evaluated herein and was restricted to clades 6-9.

Additional support for the distribution of the intact and deleted versions of nor $V$ was previously observed in different O157 strain collections. The initial screen of $100 \mathrm{O} 157$ strains recovered from multiple sources detected the 204 bp deletion in $58 \%$ of strains and a correlation with stx1 [14]. In our analysis, however, the deletion was most common among strains possessing both st $x 1$ and stx 2 representing clades 2 and 3, which indicates variation across the strain collections. This variation is not surprising given that Stx-encoding bacteriophages can integrate within multiple sites in different $E$. coli genomes [29]; hence, geographic variation could be due to the prevalence of circulating bacteriophages in certain locations. Consistent with our data, another screen of 107 distinct O157 strains confirmed the nor $V$ deletion to be restricted to clades $1-3$, while the intact nor $V$ was correlated with clades 6-8 [30]. These findings indicate that nor $V$ functionality is linked to the genetic background of a given $\mathrm{O} 157$ strain. Additionally, the intact nor $V$ was detected in a subset of 34 EHEC strains representing 10 non-O157 serotypes [31], suggesting a more widespread distribution. The association observed between clade 8 strains, which all contained an intact $n o r V$, and the presence of stx2 alone or with stx2c illustrates the importance of the $\mathrm{O} 157$ genetic background on virulence. Indeed, other strains with both an intact or deleted version of nor $V$ possess these stx subtypes either alone or in combination with st $x 1$, suggesting that other factors also contribute to enhanced virulence in clade 8 strains. Examining nor $V$ function and its relationship with virulence is therefore warranted in a larger collection of clade 8 strains.

In anaerobic conditions, we demonstrated that $\mathrm{NO}$ induces st $x 2$ expression in the clade 8 spinach outbreak strain, TW14359, resulting in a $>2$-fold increase of $s t x 2$ transcription compared to growth in the absence of NO. In aerobic conditions, however, similar stx2 transcription levels were observed in the presence and absence of NO. As the primary habitat for EHEC during infection is in the oxygen restricted intestine, it can encounter stressful conditions that may include NO produced by macrophages or endothelial and epithelial cells [32]. Our data showing increased stx2 expression in TW14359 in anaerobic conditions, which mimic the gut environment, differ from responses observed in other O157 strains. For instance, in the clade 3 O157:H7 EDL933 outbreak strain, NO contributed to a decrease in Stx2 production by repressing the SOS response via the nitrite-sensitive repressor (NsrR) that senses NO [33]. NO was also shown to reduce expression of LEEassociated virulence genes, resulting in decreased adherence to epithelial cells in vitro [34]. These results, however, differ from those generated in another study using the same strain, EDL933, which found low NO concentrations induce production of Stx2 in anaerobic conditions [35]. The same study showed that a higher concentration of NO was needed 
for Stx1 production as well as Stx2 production in aerobic conditions, highlighting the complexity of NO signaling in EHEC O157.

Since NorV was previously shown to reduce NO, we sought to investigate the relationship between NorV and stx 2 expression in the clade 8 TW14359 strain. Notably, the $\Delta$ nor $V$ null mutant showed increased expression of $s t x 2$ relative to the wild-type (WT) strain, indicating that NorV inhibits stx 2 expression in this strain. Expression of norV in trans on a plasmid in the $\Delta$ nor $V$ strain, however, failed to decrease st $x 2$ expression to levels similar to those observed for the WT strain. This failure to complement the WT phenotype is likely due to the use of ampicillin during the growth of the complemented strain since the pCR2.1-TOPO plasmid has an ampicillin resistance gene for selection. Multiple antibiotics, including ampicillin, have previously been shown to induce stx expression [36]. Contrary to our findings, Shimizu et al. [18] showed that by replacing the deleted norV copy found in the clade 3 EDL933 strain with an intact nor $V$ copy, st $x 2$ expression was increased in the presence of NO. This finding suggests that NorV induces the expression of st $x 2$ in EDL933. Moreover, another study showed that deleting norVW in the O157:H7 strain 620 was linked to a reduction in Stx production and persistence in the murine gut during infection; however, deletion of norVW in an O91:H21 strain (B2F1) had no impact on Stx production or persistence [31]. The reasons behind these phenotypic differences are not completely understood, although they are likely due to a variation in gene regulation and expression across $\mathrm{O} 157$ strains.

As part of the innate immune response to eliminate bacterial pathogens, $\mathrm{NO}$ is produced by the inducible NO synthase (iNOS) pathway within macrophages [37], which subsequently induces the bacterial SOS response. Therefore, we examined the role of norV in the survival of the O157:H7 strain TW14359 within THP-1 derived macrophages. Compared to the WT strain, the $\Delta$ nor $V$ mutant was not impaired in its ability to survive within macrophages. This finding suggests that NorV is not required for $\mathrm{NO}$ detoxification by strain TW14359 to enhance survival inside macrophages and that other factors may be necessary. Our preliminary finding that nor $V$ expression did not differ in TW14359 in the presence or absence of $\mathrm{NO}(n=2$; data not shown) provides further support for this hypothesis. This finding, however, requires confirmation and an additional set of clade 8 strains should be evaluated to better define strain-specific responses.

In addition to NorV, EHEC was shown to possess hmp and hcp encoding a flavohemoglobin (Hmp) [38] and high affinity NO reductase (Hcp) [39], respectively, which act independently to lower the toxicity of NO in different conditions [40]. Therefore, it is likely that either Hmp or Hcp assist with NO detoxification in TW14359 to promote survival inside macrophages and that one or both products could compensate for the loss of NorV activity. Indeed, NorV and Hmp were previously found to work synergistically during anaerobic growth [40]. Our results are consistent with those from another study demonstrating that survival of a nor $V$ mutant in the non-pathogenic E. coli strain MG1655 was not impaired in J774.2 mouse derived macrophages compared to the wild-type strain [41]. Similarly, no significant differences were observed in the survival of a Salmonella enterica Typhimurium strain within murine macrophages relative to the isogenic mutant lacking norV [42]. Contrary to our results, however, the number of surviving bacterial cells within murine macrophages following infection with the EHEC O157 K15 strain containing an intact nor $V$ was greater than the number following infection with a nor $V$ deficient mutant [18]. While this finding suggests that nor $V$ confers a protective advantage in resisting killing within macrophages for strain $\mathrm{K} 15$, the discrepant results are most likely due to the use of different $\mathrm{O} 157$ strains. Unlike TW14359, which belongs to clade 8 and contains an intact $n o r V$, strain $\mathrm{K} 15$ was found to represent clade 7 [30], the lineage linked to less severe symptoms in our prior study [10], despite having an intact nor $V$. There is also the possibility that the RAW264.7 mouse derived macrophage cells, which were used to examine the survival of the EHEC K15 strain after $10 \mathrm{~h}$ [18], may differ from the human derived THP-1 cells used herein. Indeed, a prior study observed key differences in protein expression profiles between mouse-derived RAW264.7 macrophages and THP-1 cells [43]. 
In summary, our study shows that $\mathrm{NO}$ induces the expression of stx 2 in anaerobic conditions in the clade 8 spinach outbreak strain, TW14359, thereby increasing its pathogenicity. With that in mind, selected inhibition of NO production in the host could become the focus of extensive pharmaceutical research in the future. We also demonstrated that NorV inhibits st $x 2$ expression in TW14359, which is the opposite of what we might expect when considering the enhanced virulence of clade 8 strains. While inhibiting Stx production is important for ensuring bacterial survival, a prior study showed that varying NO concentrations can induce different Stx production pathways [35], further highlighting the complex relationship between NO signaling and Stx regulation. These data are relevant since the level of Stx inhibition likely varies across conditions and environments encountered in the host. As we have shown that O157 strains can have variable levels of stx expression [11,44,45], it is clear that strain-specific factors can also impact transcriptional regulation. Given the importance of strain-specific differences, a more comprehensive investigation into how nor $V$ impacts stx expression in other clade 8 strain backgrounds is therefore warranted. Lastly, in contrary to data generated in another study [18], we showed that nor $V$ is not required for the survival of TW14359 within THP-1 macrophages. This finding further suggests that the mechanisms for surviving in the presence of nitrosative stress, particularly following phagocytic uptake, vary across strains and that these mechanisms are complex and may require multiple factors that differ depending on the environment.

\section{Conclusions}

Collectively, our investigation of NorV in a clade 8 strain from a 2006 multistate outbreak linked to spinach consumption suggests that it plays a role in the inhibition of Stx2 production. The specific mechanism of inhibition, however, remains unclear and requires further study.

Author Contributions: Conceptualization, R.A.S. and S.D.M.; Data curation, S.D.M.; Formal analysis, R.A.S., M.L.K. and S.D.M.; Methodology, R.A.S. and M.L.K.; Project administration, S.D.M.; Resources, S.D.M.; Supervision, S.D.M.; Validation, R.A.S., M.L.K. and S.D.M.; Visualization, R.A.S., M.L.K. and S.D.M.; Writing—original draft, R.A.S.; Writing—review and editing, M.L.K. and S.D.M. All authors have read and agreed to the published version of the manuscript.

Funding: This research was funded by the U.S. Department of Agriculture (USDA), grant number 2011-67005-30004, while salary support was provided by the USDA, grant number 2019-67017-2911, the Michigan State University Foundation, and the HHS I NIH I National Institute of Allergy and Infectious Diseases (NIAID), grant number U19AI090872.

Institutional Review Board Statement: This study was conducted in accordance with the Declaration of Helsinki and was approved by the Institutional Review Board at Michigan State University (10-736SM) and the Michigan Department of Health and Human Services (842-PHALAB).

Informed Consent Statement: Patient consent was waived due to the use of specimens and data collected for public health purposes.

Data Availability Statement: All data supporting the results can be found within the manuscript.

Acknowledgments: The authors thank Lindsey Ouellette for technical support, Pallavi Singh for helpful discussions, and James T. Rudrik and Patricia Somsel of the Michigan Department of Health and Human Services, Bureau of Laboratories for previously sharing the O157 isolates.

Conflicts of Interest: The authors declare no conflict of interest. The funders had no role in the design of the study; in the collection, analyses, or interpretation of data; in the writing of the manuscript, or in the decision to publish the results.

\section{References}

1. Karmali, M.A.; Petric, M.; Lim, C.; McKeough, P.C.; Arbus, G.S.; Lior, H. The Association between Idiopathic Hemolytic Uremic Syndrome and Infection by Verotoxin-Producing Escherichia coli. J. Infect. Dis. 1985, 151, 775-782. [CrossRef]

2. Scallan, E.; Hoekstra, R.M.; Angulo, F.J.; Tauxe, R.V.; Widdowson, M.A.; Roy, S.L.; Jones, J.L.; Griffin, P.M. Foodborne Illness Acquired in the United States-Major Pathogens. Emerg. Infect. Dis. 2011, 17, 7-15. [CrossRef] 
3. Tack, D.M.; Marder, E.P.; Griffin, P.M.; Cieslak, P.R.; Dunn, J.; Hurd, S.; Scallan, E.; Lathrop, S.; Muse, A.; Ryan, P.; et al. Preliminary Incidence and Trends of Infections with Pathogens Transmitted Commonly Through Food-Foodborne Diseases Active Surveillance Network, 10 U.S. Sites, 2015-2018. Morb. Mortal. Wkly. Rep. 2019, 68, 369-373. [CrossRef]

4. Centers for Disease Control and Prevention. In National Shiga Toxin-Producing Escherichia coli (STEC) Surveillance Annual Report, 2016; Centers for Disease Control and Prevention: Atlanta, GA, USA, 2018. Available online: www.cdc.gov/ecoli/surv2016/ index.html (accessed on 30 December 2020).

5. Strockbine, N.A.; Marques, L.R.M.; Newland, J.W.; Smith, H.W.; Holmes, R.K.; O'brien, A.D. Two Toxin-Converting Phages from Escherichia coli O157:H7 Strain 933 Encode Antigenically Distinct Toxins with Similar Biologic Activities. Infect. Immun. 1986, 53, 135-140. [CrossRef]

6. McDaniel, T.K.; Kaper, J.B. A Cloned Pathogenicity Island from Enteropathogenic Escherichia coli Confers the Attaching and Effacing Phenotype on E. coli K-12. Mol. Microbiol. 1997, 23, 399-407. [CrossRef] [PubMed]

7. Nataro, J.P.; Kaper, J.B. Diarrheagenic Escherichia coli. Clin. Microbiol. Rev. 1998, 11, 142-201. [CrossRef] [PubMed]

8. Ostroff, S.M.; Tarr, P.I.; Neill, M.A.; Lewis, J.H.; Hargrett-Bean, N.; Kobayashi, J.M. Toxin Genotypes and Plasmid Profiles as Determinants of Systemic Sequelae in Escherichia coli O157:H7 Infections. J. Infect. Dis. 1989, 160, 994-998. [CrossRef] [PubMed]

9. Orth, D.; Grif, K.; Khan, A.B.; Naim, A.; Dierich, M.P.; Würzner, R. The Shiga Toxin Genotype Rather than the Amount of Shiga Toxin or the Cytotoxicity of Shiga Toxin in vitro Correlates with the Appearance of the Hemolytic Uremic Syndrome. Diagn. Microbiol. Infect. Dis. 2007, 59, 235-242. [CrossRef] [PubMed]

10. Manning, S.D.; Motiwala, A.S.; Springman, A.C.; Qi, W.; Lacher, D.W.; Ouellette, L.M.; Mladonicky, J.M.; Somsel, P.; Rudrik, J.T.; Dietrich, S.E.; et al. Variation in Virulence among Clades of Escherichia coli O157:H7 Associated with Disease Outbreaks. Proc. Natl. Acad. Sci. USA 2008, 105, 4868-4873. [CrossRef] [PubMed]

11. Abu-Ali, G.S.; Ouellette, L.M.; Henderson, S.T.; Lacher, D.W.; Riordan, J.T.; Whittam, T.S.; Manning, S.D. Increased Adherence and Expression of Virulence Genes in a Lineage of Escherichia coli O157:H7 Commonly Associated with Human Infections. PLoS ONE 2010, 5, e10167. [CrossRef] [PubMed]

12. Henderson, S.T.; Singh, P.; Knupp, D.; Lacher, D.W.; Abu-Ali, G.S.; Rudrik, J.T.; Manning, S.D. Variability in the Occupancy of Escherichia coli O157 Integration Sites by Shiga Toxin-Encoding Prophages. Toxins 2021, 13, 433. [CrossRef] [PubMed]

13. Centers for Disease Control and Prevention. Ongoing Multistate Outbreak of Escherichia coli Serotype O157:H7 Infections Associated with Consumption of Fresh Spinach. Morb. Mortal. Wkly. Rep. 2006, 55, 1-2.

14. Kulasekara, B.R.; Jacobs, M.; Zhou, Y.; Wu, Z.; Sims, E.; Saenphimmachak, C.; Rohmer, L.; Ritchie, J.M.; Radey, M.; McKevitt, M.; et al. Analysis of the Genome of the Escherichia coli O157:H7 2006 Spinach-Associated Outbreak Isolate Indicates Candidate Genes that May Enhance Virulence. Infect. Immun. 2009, 77, 3713-3721. [CrossRef] [PubMed]

15. Gardner, A.M.; Helmick, R.A.; Gardner, P.R. Flavorubredoxin, an Inducible Catalyst for Nitric Oxide Reduction and Detoxification in Escherichia coli. J. Biol. Chem. 2002, 277, 8172-8177. [CrossRef] [PubMed]

16. Perna, N.T.; Plunkett, G.; Burland, V.; Mau, B.; Glasner, J.D.; Rose, D.J.; Mayhew, G.F.; Evans, P.S.; Gregor, J.; Kirkpatrick, H.A.; et al. Genome Sequence of Enterohaemorrhagic Escherichia coli O157:H7. Nature 2001, 409, 529-533. [CrossRef]

17. Hayashi, T.; Makino, K.; Ohnishi, M.; Kurokawa, K.; Ishii, K.; Yokoyama, K.; Han, C.G.; Ohtsubo, E.; Nakayama, K.; Murata, T.; et al. Complete Genome Sequence of Enterohemorrhagic Escherichia coli O157:H7 and Genomic Comparison with a Laboratory Strain K-12. DNA Res. 2001, 8, 11-22. [CrossRef] [PubMed]

18. Shimizu, T.; Tsutsuki, H.; Matsumoto, A.; Nakaya, H.; Noda, M. The Nitric Oxide Reductase of Enterohaemorrhagic Escherichia coli Plays an Important Role for the Survival within Macrophages. Mol. Microbiol. 2012, 85, 492-512. [CrossRef]

19. Manning, S.D.; Madera, R.T.; Schneider, W.; Dietrich, S.E.; Khalife, W.; Brown, W.; Whittam, T.S.; Somsel, P.; Rudrik, J.T. Surveillance for Shiga Toxin-Producing Escherichia coli, Michigan, 2001-2005. Emerg. Infect. Dis. 2007, 13, 318. [CrossRef]

20. Murphy, K.C.; Campellone, K.G. Lambda Red-Mediated Recombinogenic Engineering of Enterohemorrhagic and Enteropathogenic E. Coli. BMC Mol. Biol. 2003, 4, 11. [CrossRef] [PubMed]

21. Vanaja, S.K.; Bergholz, T.M.; Whittam, T.S. Characterization of the Escherichia coli O157:H7 Sakai GadE Regulon. J. Bacteriol. 2009, 191. [CrossRef]

22. Al Safadi, R.; Abu-Ali, G.S.; Sloup, R.E.; Rudrik, J.T.; Waters, C.M.; Eaton, K.A.; Manning, S.D. Correlation between in vivo Biofilm Formation and Virulence Gene Expression in Escherichia coli O104:H4. PLoS ONE. 2012, 7. [CrossRef] [PubMed]

23. Datsenko, K.A.; Wanner, B.L. One-Step Inactivation of Chromosomal Genes in Escherichia coli K-12 Using PCR Products. Proc. Natl. Acad. Sci. USA 2000, 97, 6640-6645. [CrossRef]

24. Bergholz, T.M.; Wick, L.M.; Qi, W.; Riordan, J.T.; Ouellette, L.M.; Whittam, T.S. Global Transcriptional Response of Escherichia coli O157:H7 to Growth Transitions in Glucose Minimal Medium. BMC Microbiol. 2007, 7, 97. [CrossRef] [PubMed]

25. Schmittgen, T.D.; Livak, K.J. Analyzing Real-Time PCR Data by the Comparative CT Method. Nat. Prot. 2008, 3, 1101-1108. [CrossRef]

26. Schwende, H.; Fitzke, E.; Ambs, P.; Dieter, P. Differences in the State of Differentiation of THP-1 Cells Induced by Phorbol Ester and 1,25-Dihydroxyvitamin D3. J. Leukoc. Biol. 1996, 59, 555-561. [CrossRef] [PubMed]

27. Korir, M.L.; Laut, C.; Rogers, L.M.; Plemmons, J.A.; Aronoff, D.M.; Manning, S.D. Differing Mechanisms of Surviving Phagosomal Stress among Group B Streptococcus Strains of Varying Genotypes. Virulence 2017, 8, 924-937. [CrossRef]

28. Zangari, T.; Melton-Celsa, A.R.; Panda, A.; Smith, M.A.; Tatarov, I.; de Tolla, L.; O'Brien, A.D. Enhanced Virulence of the Escherichia coli O157:H7 Spinach-Associated Outbreak Strain in Two Animal Models Is Associated with Higher Levels of Stx2 Production after Induction with Ciprofloxacin. Infect. Immun. 2014, 82, 4968-4977. [CrossRef] [PubMed] 
29. Shaikh, N.; Tarr, P.I. Escherichia coli O157:H7 Shiga Toxin-Encoding Bacteriophages: Integrations, Excisions, Truncations, and Evolutionary Implications. J. Bacteriol. 2003, 185, 3596-3605. [CrossRef]

30. Shimizu, T.; Hirai, S.; Yokoyama, E.; Ichimura, K.; Noda, M. An Evolutionary Analysis of Nitric Oxide Reductase Gene NorV in Enterohemorrhagic Escherichia coli O157. Infect. Genet. Evol. 2015, 33, 176-181. [CrossRef]

31. Gardette, M.; Daniel, J.; Loukiadis, E.; Jubelin, G. Role of the Nitric Oxide Reductase NorVW in the Survival and Virulence of Enterohaemorrhagic Escherichia coli during Infection. Pathogens 2020, 9, 683. [CrossRef]

32. Flint, A.; Butcher, J.; Stintzi, A. Stress Responses, Adaptation, and Virulence of Bacterial Pathogens during Host Gastrointestinal Colonization. Microbiol. Spectr. 2016, 4. [CrossRef]

33. Vareille, M.; de Sablet, T.; Hindré, T.; Martin, C.; Gobert, A.P. Nitric Oxide Inhibits Shiga-Toxin Synthesis by Enterohemorrhagic Escherichia coli. Proc. Natl. Acad. Sci. USA 2007, 104, 10199-10204. [CrossRef] [PubMed]

34. Branchu, P.; Matrat, S.; Vareille, M.; Garrivier, A.; Durand, A.; Crépin, S.; Harel, J.; Jubelin, G.; Gobert, A.P. NsrR, GadE, and GadX Interplay in Repressing Expression of the Escherichia coli O157:H7 LEE Pathogenicity Island in Response to Nitric Oxide. PLoS Pathog. 2014, 10, e1003874. [CrossRef] [PubMed]

35. Ichimura, K.; Shimizu, T.; Matsumoto, A.; Hirai, S.; Yokoyama, E.; Takeuchi, H.; Yahiro, K.; Noda, M. Nitric Oxide-Enhanced Shiga Toxin Production Was Regulated by Fur and RecA in Enterohemorrhagic Escherichia coli O157. Microbiol. Open 2017, 6, e00461. [CrossRef]

36. McGannon, C.M.; Fuller, C.A.; Weiss, A.A. Different Classes of Antibiotics Differentially Influence Shiga Toxin Production. Antimicrob. Agents Chemother. 2010, 54, 3790-3798. [CrossRef] [PubMed]

37. MacMicking, J.; Xie, Q.W.; Nathan, C. Nitric Oxide and Macrophage Function. Annu. Rev. Immunol. 1997, 15, 323-350. [CrossRef]

38. Gardner, A.M.; Gardner, P.R. Flavohemoglobin Detoxifies Nitric Oxide in Aerobic, but Not Anaerobic, Escherichia coli: Evidence for a Novel Inducible Anaerobic Nitric Oxide-Scavenging Activity. J. Biol. Chem. 2002, 277. [CrossRef]

39. Wang, J.; Vine, C.E.; Balasiny, B.K.; Rizk, J.; Bradley, C.L.; Tinajero-Trejo, M.; Poole, R.K.; Bergaust, L.L.; Bakken, L.R.; Cole, J.A. The Roles of the Hybrid Cluster Protein, Hcp and Its Reductase, Hcr, in High Affinity Nitric Oxide Reduction That Protects Anaerobic Cultures of Escherichia coli against Nitrosative Stress. Mol. Microbiol. 2016, 100. [CrossRef]

40. Shimizu, T.; Matsumoto, A.; Noda, M. Cooperative Roles of Nitric Oxide-Metabolizing Enzymes to Counteract Nitrosative Stress in Enterohemorrhagic Escherichia coli. Infect. Immun. 2019, 87, e00334-19. [CrossRef]

41. Pullan, S.T.; Gidley, M.D.; Jones, R.A.; Barrett, J.; Stevanin, T.M.; Read, R.C.; Green, J.; Poole, R.K. Nitric Oxide in ChemostatCultured Escherichia coli Is Sensed by Fnr and Other Global Regulators: Unaltered Methionine Biosynthesis Indicates Lack of S Nitrosation. J. Bacteriol. 2007, 189, 1845-1855. [CrossRef]

42. Bang, I.S.; Liu, L.; Vazquez-Torres, A.; Crouch, M.L.; Stamler, J.S.; Fang, F.C. Maintenance of Nitric Oxide and Redox Homeostasis by the Salmonella Flavohemoglobin Hmp. J. Biol. Chem. 2006, 281, 28039-28047. [CrossRef]

43. Li, P.; Hao, Z.; Wu, J.; Ma, C.; Xu, Y.; Li, J.; Lan, R.; Zhu, B.; Ren, P.; Fan, D.; et al. Comparative Proteomic Analysis of Polarized Human THP-1 and Mouse RAW264.7 Macrophages. Front. Immunol. 2021, 12, 700009. [CrossRef] [PubMed]

44. Abu-Ali, G.S.; Ouellette, L.M.; Henderson, S.T.; Whittam, T.S.; Manning, S.D. Differences in Adherence and Virulence Gene Expression between Two Outbreak Strains of Enterohaemorrhagic Escherichia coli O157:H7. Microbiology 2010, 156, 408-419. [CrossRef] [PubMed]

45. Neupane, M.; Abu-Ali, G.S.; Mitra, A.; Lacher, D.W.; Manning, S.D.; Riordan, J.T. Shiga Toxin 2 Overexpression in Escherichia coli O157:H7 Strains Associated with Severe Human Disease. Microb. Pathog. 2011, 51, 466-470. [CrossRef] [PubMed] 